\title{
Relationship between Shehu Transform with Some other Integral Transform
}

\author{
Mulugeta Andualem, Atinafu Asfaw
}

\begin{abstract}
Integral transformations have been successfully used for almost two centuries in solving many problems in applied mathematics, mathematical physics, and engineering science. Shehu transform is new integral transform type which is convenient mathematical methods for solving advance problems of engineering and sciences which are mathematically expressed in terms of differential equations, system of differential equations, partial differential equations,
\end{abstract}

\section{INTRODUCTION}

Many problems in engineering and science can be formulated in terms of differential equations. The ordinary differential equations arise in many areas of Mathematics, as well as in Sciences and Engineering.

\section{SHEHU TRANSFORM}

Definition: A new transform called the Shehu transform of the function $f(t)$ belonging to a class $A$, where

$$
A=\left\{\begin{array}{c}
f(t): \exists N, \eta_{1}, \eta_{2}>0,|f(t)| \\
<N e^{\frac{|t|}{\eta_{i}}, \text { if } t \in(-1)^{i} \times[0 \infty)}
\end{array}\right\}
$$

Where $f(t)$ defined by $\mathbb{S}[f(t)]$ and is given by:

$$
\begin{aligned}
\mathbb{S}\{f(t)\} & =W(s, u) \\
& =\int_{0}^{\infty} e^{\left(\frac{-s t}{u}\right)} f(t) d t
\end{aligned}
$$

\section{ZZ TRANSFORM}

Let $f(t)$ be a function defined for all $t \geq 0$. The ZZ transform of $f(t)$ is the function $Z(u, s)$ defined by:

$$
\begin{aligned}
Z(u, s) & =H\{f(t)\} \\
& =s \int_{0}^{\infty} f(u t) e^{-s t} d t
\end{aligned}
$$

\section{MOHAND TRANSFORM}

integral equations, system of integral equations, partial integro-differential equations and integro differential equations. In this study, we discussed the relationship between this new integral transform with other some integral transforms.

Keywords: Shehu transform, ZZ transform, Mohand transform, Laplace transform, Sawi transform, Mahgoub transform.

In order to solve the certain ordinary differential equations integral transforms are widely used. In this article we have construct the relation between Shehu transform and some other integral transforms which helps us to use Shehu transform simply in solving differential equations.

Mohand transform of the function $f(t) t \geq 0$ is given by:

$$
\begin{aligned}
M\{f(t)\} & =H(s)_{\infty} \\
& =s^{2} \int_{0}^{\infty} f(t) e^{-s t} d t
\end{aligned}
$$

\section{LAPLACE TRANSFORM}

The Laplace transform of the function $f(t) t \geq 0$ is given by:

$$
\begin{aligned}
L\{f(t)\} & =F(s) \\
& =\int_{0}^{\infty} f(t) e^{-s t} d t
\end{aligned}
$$

\section{MAHGOUB TRANSFORM}

Mahgoub (Laplace-Carson) transform of the function $f(t) t \geq 0$ is given by:

$$
\begin{gathered}
M_{*}\{f(t)\}=s \int_{0}^{\infty} f(t) e^{-s t} d t \\
0<k_{1} \leq s \leq k_{2}
\end{gathered}
$$

\section{SAWI TRANSFORM}

Sawi transform of the function $f(t) t \geq 0$ is given by: 


$$
\begin{gathered}
M_{s}\{f(t)\}=\frac{1}{s^{2}} \int_{0}^{\infty} f(t) e^{\frac{-t}{s}} d t \\
0<k_{1} \leq k_{2}
\end{gathered}
$$

\section{A. Connection between Shehu transform and ZZ transform}

In this section we show that Shehu transform is theoretical dual of $\mathrm{ZZ}$ transform and the dual relation is given by the following relation:

Theorem 1.1: Let $f(t) \in A$ and if the Shehu transform and ZZ transform of $f(t)$ are $W(s, u)$ and $Z(u, s)$ respectively then

$$
Z(u, s)=\frac{s}{u} W(s, u)
$$

And

$$
\frac{u}{s} Z(u, s)=W(s, u)
$$

Proof: From (1.2) we have

$$
Z(u, s)=s \int_{0}^{\infty} f(u t) e^{-s t} d t
$$

Let $w=u t \Rightarrow d t=\frac{d w}{u}$ from the above equation we have

$$
\begin{aligned}
Z(u, s)=s \int_{0}^{\infty} f(u t) e^{-s t} d t \\
\Rightarrow Z(u, s)=s \int_{0}^{\infty} f(w) e^{\frac{-s w}{u}} \frac{d w}{u} \\
\Rightarrow Z(u, s)=\frac{s}{u} \int_{0}^{\infty} f(w) e^{\frac{-s w}{u}} d w \\
\Rightarrow Z(u, s)=\frac{s}{u} W(s, u)
\end{aligned}
$$

Hence the proof is completed

Now, to drive (1.8, from (1.1) we have:

$$
\mathbb{S}\{f(t)\}=W(s, u)=\int_{0}^{\infty} e^{\left(\frac{-s t}{u}\right)} f(t) d t
$$

$$
\Rightarrow W(s, u)=\frac{u}{s}\left(\frac{s}{u} \int_{0}^{\infty} f(w) e^{\frac{-s w}{u}} d w\right)
$$

Since, from (1.2)

$$
\begin{aligned}
\left(\frac{s}{u} \int_{0}^{\infty} f(w) e^{\frac{-s w}{u}} d w\right) & =Z(u, s) \\
\Rightarrow W(s, u) & =\frac{u}{s} Z(u, s)
\end{aligned}
$$

Hence the proof of (1.8) is completed

Table 1: The relationship between Shehu transform and $\mathrm{ZZ}$ transform on some common functions

\begin{tabular}{|c|c|c|c|}
\hline$f(t)$ & $\begin{array}{c}\mathbb{S}\{f(t)\} \\
=W(s, u)\end{array}$ & $\begin{array}{c}H\{f(t)\} \\
=Z(u, s)\end{array}$ & $\begin{array}{l}\frac{u}{s} Z(u, s) \\
=W(s, u)\end{array}$ \\
\hline 1 & $\frac{u}{s}$ & 1 & $\frac{u}{s}$ \\
\hline$t$ & $\frac{u^{2}}{s^{2}}$ & $\frac{u}{s}$ & $\frac{u^{2}}{s^{2}}$ \\
\hline$t^{2}$ & $\frac{2 ! u^{3}}{s^{3}}$ & $\frac{2 ! u^{2}}{s^{2}}$ & $\frac{2 ! u^{3}}{s^{3}}$ \\
\hline$t^{n}$ & $\frac{n ! u^{n+1}}{s^{n+1}}$ & $\frac{n ! u^{n}}{s^{n}}$ & $\frac{n ! u^{n+1}}{s^{n+1}}$ \\
\hline$e^{a t}$ & $\frac{u}{s-a u}$ & $\frac{s}{s-a u}$ & $\frac{u-a u}{s^{2}}$ \\
\hline $\cos (a t)$ & $\frac{u s}{s^{2}+\alpha^{2} u^{2}}$ & $\frac{s^{2}}{s^{2}+\alpha^{2} u^{2}}$ & $\frac{a u s}{s^{2}+\alpha^{2} u^{2}}$ \\
\hline $\sin (a t)$ & $\frac{\alpha u^{2}}{s^{2}+\alpha^{2} u^{2}}$ & $\frac{\alpha u^{2}}{s^{2}+(a u)^{2}}$ & $\frac{s^{2}+\alpha^{2} u^{2}}{s^{2}}$ \\
\hline
\end{tabular}

\section{B. Connection between Shehu transform and Laplace transform}

Theorem 1.2: Let $f(t) \in A$ and $t \geq 0$ if the Shehu transform and Laplace transform of $f(t)$ are $W(s, u)$ and $F(s)$ respectively then

$$
W(s, u)=F\left(\frac{s}{u}\right)
$$

Proof: Since $W(s, u)=\frac{u}{s} Z(u, s)$

$$
\Rightarrow W(s, u)=\frac{u}{s}\left(s \int_{0}^{\infty} f(u t) e^{-s t} d t\right)
$$




$$
\begin{gathered}
\Rightarrow W(s, u)=\frac{u}{s}\left(s \int_{0}^{\infty} f(u t) e^{-s t} d t\right) \\
\Rightarrow W(s, u)=u \int_{0}^{\infty} f(u t) e^{-s t} d t
\end{gathered}
$$

Put $w=u t \Rightarrow \frac{d w}{u}=d t$ in the above equation, we have

$$
\begin{gathered}
\Rightarrow W(s, u)=u \int_{0}^{\infty} f(t) e^{\frac{-s w}{u}} \frac{d w}{u} \\
\Rightarrow W(s, u)=\int_{0}^{\infty} f(t) e^{\frac{-s w}{u}} d w=F\left(\frac{s}{u}\right)
\end{gathered}
$$

Therefore $W(s, u)=F\left(\frac{s}{u}\right)$

Hence the proof is completed

Table 2: The relationship between Shehu transform and Laplace transform of some common functions.

\begin{tabular}{|c|c|c|c|}
\hline$f(t)$ & $\begin{array}{c}S\{f(t)\} \\
=W(s, u)\end{array}$ & $\begin{array}{c}\mathcal{L}\{f(t)\} \\
=F(s)\end{array}$ & $\begin{array}{c}F\left(\frac{s}{u}\right) \\
=W(s, u)\end{array}$ \\
\hline 1 & $\frac{u}{s}$ & $\frac{1}{s}$ & $\frac{u}{s}$ \\
\hline$t$ & $\frac{u^{2}}{s^{2}}$ & $\frac{1}{s^{2}}$ & $\frac{u^{2}}{s^{2}}$ \\
\hline$t^{2}$ & $\frac{2 ! u^{3}}{s^{3}}$ & $\frac{2 !}{s^{3}}$ & $\frac{2 ! u^{3}}{s^{3}}$ \\
\hline$t^{n}$ & $\frac{n ! u^{n+1}}{s^{n+1}}$ & $\frac{n !}{s^{n+1}}$ & $\frac{n ! u^{n+1}}{s^{n+1}}$ \\
\hline$e^{a t}$ & $\frac{u}{s-a u}$ & $\frac{1}{s-a}$ & $\frac{u-a u}{s-s^{3}}$ \\
\hline $\cos (a t)$ & $\frac{u s}{s^{2}+\alpha^{2} u^{2}}$ & $\frac{s^{2}}{s^{2}+\alpha^{2}}$ & $\frac{u s^{2}+\alpha^{2} u^{2}}{s^{2}}$ \\
\hline $\sin (a t)$ & $\frac{\alpha u^{2}}{s^{2}+\alpha^{2} u^{2}}$ & $\frac{\alpha s^{2}}{s^{2}+\alpha^{2}}$ & $\frac{\alpha u^{2}}{s^{2}+\alpha^{2} u^{2}}$ \\
\hline
\end{tabular}

\section{Connection between Shehu transform and Mohand transform}

Theorem 1.3: Let $f(t) \in A$ and $t \geq 0$, if the Shehu transform and Mohand transform of $f(t)$ are $W(s, u)$ and $H(s)$ respectively then

$$
\frac{u^{2}}{s^{2}} H\left(\frac{s}{u}\right)=W(s, u)
$$

And

$$
H\left(\frac{s}{u}\right)=\frac{s^{2}}{u^{2}} W(s, u)
$$

Proof: Since, $W(s, u)=F\left(\frac{s}{u}\right)$

$$
\begin{gathered}
M\{f(t)\}=H(s)=s^{2} \int_{0}^{\infty} f(t) e^{-s t} d t \\
\Rightarrow M\{f(t)\}=s^{2}\left(\int_{0}^{\infty} f(t) e^{-s t} d t\right) \\
\Rightarrow M\{f(t)\}=H(s)=s^{2} F(s)
\end{gathered}
$$

Now, if we substitute $s \rightarrow \frac{s}{u}$

$$
\begin{aligned}
& \Rightarrow M\{f(t)\}=H\left(\frac{s}{u}\right)=\left(\frac{s}{u}\right)^{2} F\left(\frac{s}{u}\right) \\
& \Rightarrow \frac{u^{2}}{s^{2}} M\{f(t)\}=\frac{u^{2}}{s^{2}} H\left(\frac{s}{u}\right)=F\left(\frac{s}{u}\right)
\end{aligned}
$$

But, from (1.9) $W(s, u)=F\left(\frac{s}{u}\right)$ in the above equation we have

$$
\Rightarrow \frac{u^{2}}{s^{2}} H\left(\frac{s}{u}\right)=W(s, u)
$$

Hence the proof is completed

Consequently, to drive (1.11)

$$
\Rightarrow \frac{u^{2}}{s^{2}} H(s)=W(s, u)
$$

Now, multiply the above equation by $\frac{s^{2}}{u^{2}}$ both sides, we have

$$
\Rightarrow H\left(\frac{s}{u}\right)=\frac{s^{2}}{u^{2}} W(s, u)
$$

Hence the proof is completed.

Table 3: The relationship between Shehu transform and Mohand transform of some common functions

\begin{tabular}{|c|c|c|c|}
\hline$f(t)$ & $\begin{array}{l}\mathbb{S}\{f(t)\} \\
=W(s, u)\end{array}$ & $\begin{array}{l}M\{f(t)\} \\
=H(s)\end{array}$ & $\begin{array}{c}\frac{u^{2}}{s^{2}} H\left(\frac{s}{u}\right) \\
=W(s, u)\end{array}$ \\
\hline 1 & $\frac{u}{s}$ & $s$ & $\frac{u}{s}$ \\
\hline
\end{tabular}




\begin{tabular}{|c|c|c|c|}
\hline$t$ & $\frac{u^{2}}{s^{2}}$ & 1 & $\frac{u^{2}}{s^{2}}$ \\
\hline$t^{2}$ & $\frac{2 ! u^{3}}{s^{3}}$ & $\frac{2 !}{s}$ & $\frac{2 ! u^{3}}{s^{3}}$ \\
\hline$t^{n}$ & $\frac{n ! u^{n+1}}{s^{n+1}}$ & $\frac{n !}{s^{n-1}}$ & $\frac{n ! u^{n+1}}{s^{n+1}}$ \\
\hline$e^{a t}$ & $\frac{u}{s-a u}$ & $\frac{s^{2}}{s-a}$ & $\frac{u}{s-a u}$ \\
\hline $\cos (a t)$ & $\frac{u s}{s^{2}+\alpha^{2} u^{2}}$ & $\frac{s^{3}}{s^{2}+\alpha^{2}}$ & $\frac{u s}{s^{2}+\alpha^{2} u^{2}}$ \\
\hline $\sin (a t)$ & $\frac{\alpha u^{2}}{s^{2}+\alpha^{2} u^{2}}$ & $\frac{\alpha s^{2}}{s^{2}+\alpha^{2}}$ & $\frac{\alpha u^{2}}{s^{2}+\alpha^{2} u^{2}}$ \\
\hline
\end{tabular}

\section{Connection between Shehu transform and Mahgoub transform}

Theorem 1.4: Let $f(t) \in A$ and $t \geq 0$, if the Shehu transform and Mahgoub transform of $f(t)$ are $W(s, u)$ and $G(s)$ respectively then

$$
\frac{s}{u} W(s, u)=G\left(\frac{s}{u}\right)
$$

And

$$
W(s, u)=\frac{u}{s} G\left(\frac{s}{u}\right)
$$

Proof: From (1.9) we have

$$
\begin{gathered}
M_{*}\{f(t)\}=s \int_{0}^{\infty} f(t) e^{-s t} d t \\
\Rightarrow M_{*}\{f(t)\}=s\left(\int_{0}^{\infty} f(t) e^{-s t} d t\right) \\
\Rightarrow M_{*}\{f(t)\}=G(s)=s F(s)
\end{gathered}
$$

Now, if we substitute $s \rightarrow \frac{s}{u}$

$$
\Longrightarrow M_{*}\{f(t)\}=G\left(\frac{s}{u}\right)=\frac{s}{u} F\left(\frac{s}{u}\right)
$$

Since from (1.9) $W(s, u)=F\left(\frac{s}{u}\right)$

$$
\Rightarrow G\left(\frac{s}{u}\right)=M_{*}\{f(t)\}=\frac{s}{u} W(s, u)
$$

Therefore, $G\left(\frac{s}{u}\right)=\frac{s}{u} W(s, u)$. Hence, the proof of (1.12) is completed
Now, multiply the above equation by $\frac{u}{s}$ both sides, we have:

$$
\Rightarrow W(s, u)=\frac{u}{s} G\left(\frac{s}{u}\right)
$$

Hence the proof of (1.13) is completed.

Table 4: The relationship between Shehu transform and Mohgoub transform of some common functions

\begin{tabular}{|c|c|c|c|}
\hline$f(t)$ & $\begin{array}{c}S\{f(t)\} \\
=W(s, u)\end{array}$ & $\begin{array}{c}M_{*}\{f(t)\} \\
=G(s)\end{array}$ & $\begin{array}{l}\frac{u}{s} G\left(\frac{s}{u}\right) \\
=W(s, u)\end{array}$ \\
\hline 1 & $\frac{u}{s}$ & 1 & $\frac{u}{s}$ \\
\hline$t$ & $\frac{u^{2}}{s^{2}}$ & $\frac{1}{s}$ & $\frac{u^{2}}{s^{2}}$ \\
\hline$t^{2}$ & $\frac{2 ! u^{3}}{s^{3}}$ & $\frac{2 !}{s^{2}}$ & $\frac{2 ! u^{3}}{s^{3}}$ \\
\hline$t^{n}$ & $\frac{n ! u^{n+1}}{s^{n+1}}$ & $\frac{n !}{s^{n}}$ & $\frac{n ! u^{n+1}}{s^{n+1}}$ \\
\hline$e^{a t}$ & $\frac{u}{s-a u}$ & $\frac{s}{s-a}$ & $\frac{u-a u}{s-s^{2}}$ \\
\hline $\cos (a t)$ & $\frac{u s}{s^{2}+\alpha^{2} u^{2}}$ & $\frac{s^{2}+\alpha^{2}}{s^{2}}$ & $\frac{\alpha s}{s^{2}+\alpha^{2} u^{2}}$ \\
\hline $\sin (a t)$ & $\frac{\alpha u^{2}}{s^{2}+\alpha^{2} u^{2}}$ & $\frac{s^{2}+\alpha^{2}}{s^{2}+\alpha^{2} u^{2}}$ \\
\hline
\end{tabular}

\section{E. Connection between Shehu transform and Sawi transform}

Theorem 1.5: Let $f(t) \in A$ and $t \geq 0$, if the Shehu transform and Sawi transform of $f(t)$ are $W(s, u)$ and $J(s)$ respectively then

$$
J\left(\frac{s}{u}\right)=\left(\frac{u}{s}\right)^{2} W(s, u)
$$

Proof: From equation (1.6) we have

$$
\begin{aligned}
M_{s}\{f(t)\}=J(s)=\frac{1}{s^{2}} \int_{0}^{\infty} f(t) e^{\frac{-t}{s}} d t \\
\Rightarrow M_{s}\{f(t)\}=J(s)=\left(\frac{1}{s}\right)^{2} \int_{0}^{\infty} f(t) e^{\frac{-t}{s}} d t \\
\Rightarrow M_{s}\{f(t)\}=J(s)=\left(\frac{1}{s}\right)^{2}\left(\int_{0}^{\infty} f(t) e^{\frac{-t}{s}} d t\right)
\end{aligned}
$$




\section{E-ISSN: 2321-9637}

\section{Available online at www.ijrat.org}

$$
\Rightarrow M_{s}\{f(t)\}=J(s)=\left(\frac{1}{s}\right)^{2} F\left(\frac{1}{s}\right)
$$

Now, if we substitute $s \rightarrow \frac{u}{s}$

$$
\begin{gathered}
\Rightarrow M_{s}\{f(t)\}=J\left(\frac{u}{s}\right)=\left(\frac{s}{u}\right)^{2} F\left(\frac{s}{u}\right)=\left(\frac{s}{u}\right)^{2} W(s, u) \\
\Rightarrow M_{s}\{f(t)\}=J\left(\frac{s}{u}\right)=\left(\frac{s}{u}\right)^{2} W(s, u)
\end{gathered}
$$

Hence, the proof is completed.

Table 5: The relationship between Shehu transform and Sawi transform of some common functions

\begin{tabular}{|c|c|c|c|}
\hline$f(t)$ & $\begin{array}{c}S\{f(t)\} \\
=W(s, u)\end{array}$ & $\begin{array}{c}M_{s}\{f(t)\} \\
=J(s)\end{array}$ & $\begin{array}{c}\frac{u^{2}}{s^{2}} J\left(\frac{s}{u}\right) \\
=W(s, u)\end{array}$ \\
\hline 1 & $\frac{u}{s}$ & $\frac{1}{s}$ & $\frac{u}{s}$ \\
\hline$t$ & $\frac{u^{2}}{s^{2}}$ & 1 & $\frac{u^{2}}{s^{2}}$ \\
\hline$t^{2}$ & $\frac{2 ! u^{3}}{s^{3}}$ & $2 ! s$ & $\frac{2 ! u^{3}}{s^{3}}$ \\
\hline$t^{n}$ & $\frac{n ! u^{n+1}}{s^{n+1}}$ & $n ! s^{n-1}$ & $\frac{n ! u^{n+1}}{s^{n+1}}$ \\
\hline$e^{a t}$ & $\frac{s}{s-a u}$ & $\frac{1}{s(1-a s)}$ & $\frac{1}{s-a u}$ \\
\hline $\cos (a t)$ & $\frac{u s}{s^{2}+\alpha^{2} u^{2}}$ & $\frac{1}{s\left(1+s^{2} \alpha^{2}\right)}$ & $\frac{u s}{s^{2}+\alpha^{2} u^{2}}$ \\
\hline $\sin (a t)$ & $\frac{\alpha u^{2}}{s^{2}+\alpha^{2} u^{2}}$ & $\frac{\alpha}{1++\alpha^{2} s^{2}}$ & $\frac{\alpha u^{2}}{s^{2}+\alpha^{2} u^{2}}$ \\
\hline
\end{tabular}

\section{CONCLUSION}

In this paper, we have successfully discussed the relationship between Shehu transform and some other integral transforms. We have also used tabular representation of Shehu transform and some other integral transform on some common functions to show the connection between Shehu transform and some other integral transform namely, ZZ transform, Mohand transform, Laplace transform, Sawi transform, Mahgoub transform.

\section{REFERENCES}

[1] Aggarwal, S., Gupta, A.R., Singh, D.P., Asthana, N. and Kumar, N., "Application of Laplace transform for solving population growth and decay problems", International Journal of Latest Technology in Engineering, Management \& Applied Science, 7(9), 141-145, 2018

[2] Chauhan, R. and Aggarwal, S., "Solution of linear partial integro-differential equations using Mahgoub transform", Periodic Research, 7(1), 28-31, 2018

[3] Sadikali Latif Shaikh, "Introducing a new Integral Transform Sadik Transform", American International Journal of Research in Science, Technology, 22(1) 100-103, 2018

[4] Sudhanshu Aggarwa, Nidhi Sharma, Raman Chauhan, "Applications of Kamal Transform for solving Volterra integral equation of first kind", International Journal of Research in Advent Technology, vol-6.No.8 ISSN: 23219637, Aug 2018

[5] Yechan Song, Hwajoon Kim, "The solution of Volterra Integral equation of Second kind by using the Elzaki Transform", Applied Mathematical Science, vol 8, No. 11, 525- 530, 2014

[6] Mahgoub, Mohand M. Abdelrahim , "The new integral transform Sawi Transform", Advances in Theoretical and Applied Mathematics, Vol. 14, No. 1, pp. 81-87, 2019

[7] Singh, G.P. and Aggarwal, S., "Sawi transform for population growth and decay problems", International Journal of Latest Technology in Engineering, Management \& Applied Science, Vol. 8, No. 8, pp. 157-162, August 2019.

[8] Lokenath Debnath and Bhatta, D., "Integral transforms and their applications", Second edition, Chapman \& Hall/CRC, 2006.

\section{AUTHORS PROFILE}

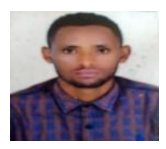

Mr. Mulugeta Andualem Abate, Lecturer, Department of Mathematics, College of Natural and Computational Science, Bonga University, Ethiopia.

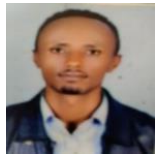

Mr. Atinafu Asfaw Yesho, Lecturer, Department of Mathematics, College of Natural and Computational Science, Bonga University, Ethiopia. 